\title{
Avaliação da função da musculatura do assoalho pélvico em pacientes com Síndrome Pós-Poliomielite
}

\author{
Evaluation of the function of pelvic floor muscles in patients \\ with Post-Polio Syndrome
}

\author{
Mirca Christina da Silva Batista ${ }^{1}$, Sissy Veloso Fontes ${ }^{2}$, \\ Acary Souza Bulle Oliveira ${ }^{3}$
}

\begin{abstract}
1.Fisioterapeuta, Especialista Fisiologia do Exercício e Sexualidade Humana, Mestre em ciências pela Universidade Federal de São Paulo (UNIFESP), São Paulo-SP, Brasil.

2.Fisioterapeuta, Psicóloga, Professora de Educação Física, Doutora em Ciências/Neurologia; Professora Afiliada do Departamento de Neurologia e Neurocirurgia da UNIFESP, São Paulo-SP, Brasil. Paulo-SP, Brasil.

3.Médico Neurologista, Pós-doutor em Neurologia, Professor Afiliado do Departamento de Neurologia e Neurocirurgia da UNIFESP, São Paulo-SP, Brasil.
\end{abstract}

\section{Resumo}

Objetivo. O estudo teve como objetivos, identificar as condições morfofuncionais da musculatura do assoalho pélvico (AP) em 78 pacientes com Síndrome pós-poliomielite (SPP), investigar as queixas relacionadas ao sistema urinário, fecal e sexual/ginecológico e correlacioná-las com a gravidade e tempo de manifestação da SPP. Assim como investigar o protocolo de avaliação fisioterapêutica para identificar disfunções do AP. Método. Foi realizada a avaliação fisioterapêutica, com anamnese e perguntas genéricas a respeito da existência de queixas relativas aos sistemas urinário, fecal e sexual/ginecológico (queixas espontâneas) e inquirições específicas para cada um dos sistemas (queixas inquiridas) e exame clínico específico (inspeção visual, palpação do AP, testes de Avaliação Funcional do Assoalho Pélvico e Manometria do AP). Resultados. Observou-se que os pacientes tinham dificuldade em relatar espontaneamente suas queixas num índice percentual apurado de $61,8 \%$ das mulheres e $30,4 \%$ dos homens. Porém, quando esclarecidas aos pacientes informações pertinentes ao AP, e realizadas investigações dirigidas, os índices passaram a $92,7 \%$ das mulheres e $87 \%$ dos homens. Conclusão. Pacientes com SPP apresentam comprometimento da musculatura do $\mathrm{AP}$, sendo que quanto maior for a fraqueza e o tempo de manifestação da síndrome pós-poliomielite maior é a quantidade de sintomas. Faz-se necessária investigação mais dirigida.

Unitermos. Síndrome Pós-Poliomielite; Assoalho Pélvico; Anamnese; Fisioterapia; Semiologia Cinesiológica Funcional.

\begin{abstract}
Objective. The objective of the study was to identify the morphofunctional conditions of the pelvic floor muscles (AP) in 78 patients with post-polio syndrome (PPS), to investigate the complaints related to the urinary, fecal and sexual/gynecological system and to correlate them with the severity, and time of manifestation of PPS. As well as investigating the physical therapy evaluation protocol to identify AP dysfunctions. Method. Physical therapy evaluation was performed, with anamnesis and general questions regarding the existence of complaints related to urinary, fecal and sexual/gynecological systems (spontaneous complaints) and specific inquiries for each system (complaints) and specific clinical examination (visual inspection). AP palpation, Pelvic Floor Functional Assessment tests and AP Manometry). Results. It was observed that patients had difficulty spontaneously reporting their complaints in an accurate percentage rate of $61.8 \%$ of women and $30.4 \%$ of men. However, when patients were informed about pertinent AP, and directed investigations were performed, the rates increased to $92.7 \%$ of women and $87 \%$ of men. Conclusion. Patients with PPS present impairment of the AP muscles, the greater the weakness and the
\end{abstract}


time of onset of post-polio syndrome, the greater the number of symptoms. More targeted research is needed.

Keywords. Post-Polio Syndrome; Pelvic Floor; Anamnesis; Physical Therapy; Functional Kinesiological Semiology.

Trabalho realizado na Disciplina de Neurologia Clínica do Departamento de Neurologia e Neurocirurgia da Universidade Federal de São Paulo/Escola Paulista de Medicina, São Paulo-SP, Brasil.

\section{INTRODUÇÃO}

A Síndrome Pós-poliomielite (SPP) foi descrita pela primeira vez em 1875, na França, por Raymond e Charcot ${ }^{1}$. Posteriormente, Carrier, Cornil e Lepine envolveram-se nos estudos relativos a essa síndrome, que ocorre em indivíduos que foram anteriormente acometidos pelos males da poliomielite paralítica aguda.

A SPP refere-se a manifestações de sintomas neuromusculares em pacientes que sofreram crise aguda da poliomielite paralítica, há muitos anos ${ }^{2,3}$. Encontra-se na categoria das doenças do neurônio motor ${ }^{4}$. Suas causas não foram ainda plenamente identificadas, o que importa em dificuldades na elaboração do diagnóstico e no gerenciamento dessa síndrome. As mais aceitas dentre essas causas são a do overuse ou supertreinamento ${ }^{5,6}$ e as do overuse associadas à idade e ao desuso ${ }^{7-9}$.

$\mathrm{Na}$ infecção aguda da poliomielite paralítica o vírus invade o Sistema Nervoso Central (SNC), causando lesão parcial ou total das células do corno anterior da medula e denervação de algumas unidades motoras. Devido ao 
mecanismo fisiológico neural, ocorre brotamento axonal com reinervação das fibras anteriormente denervadas. Esse processo compensatório está diretamente relacionado com o número de neurônios preservados. Após o período de recuperação, segue-se um período de latência, conhecido como platô de estabilidade. Na fase subsequente, devido à exacerbada solicitação metabólica das unidades motoras gigantes, há início de falência dos neurônios motores principalmente das porções distais do axônio, levando a uma nova denervação, conhecida como nova fraqueza muscular ${ }^{8}$.

A nova fraqueza muscular é o principal sintoma da SPP. Combinada a outros sintomas, leva à deterioração da habilidade funcional do indivíduo ${ }^{9,10}$. Outros sintomas observados na literatura médica são: fadiga muscular ${ }^{11}$; dificuldade progressiva do controle motor3; fraqueza geral; dores muscular e articular ${ }^{9}$.

Muito preocupantes são os achados de fraqueza progressiva nos músculos aparentemente não afetados pela poliomielite paralítica, com gradativo declínio da força neuromuscular e, em especial, da força e endurance ${ }^{12}$. Acrescente-se que atenção especial tem sido dada à diminuição da performance dos músculos esqueléticos, sobretudo os dos membros inferiores (MMII). No entanto, poucas informações a respeito dos sintomas relacionados ao comprometimento da musculatura do AP, em pacientes com doença neurológica, como a poliomielite paralítica ou à SPP, têm sido registradas na literatura ${ }^{13-16}$, contrapondo à 
ampla literatura médica encontrada, sobre funções e disfunções da musculatura do $A P^{17-22}$.

Os músculos do AP são responsáveis pelos controles esfincterianos, uretral e fecal, e pela suspensão e sustentação dos órgãos pélvicos, além de desempenharem importante papel na atividade sexual ${ }^{23}$.

Os poucos trabalhos encontrados, que estudaram a musculatura do AP e poliomielite ou SPP, não produziram análise consistente sobre as relações com os sistemas urinário, fecal e sexual/ginecológico. Foram baseados em questionários, em que muitos pacientes omitiram informações, que, na verdade, deveriam ser colhidas em uma investigação dirigida ${ }^{13,14}$.

A apresentação tímida de queixas (sintomas), relativos à musculatura do assoalho pélvico, de pacientes com SPP durante o exame médico no Setor de Investigação de Doenças Neuromusculares da UNIFESP, segundo a investigação na prática clínica dos autores desse estudo, motivou a realização desse trabalho, inédito na área de reabilitação neurológica.

Sendo assim o objetivo desse estudo foi investigar as condições morfofuncionais da musculatura do AP de pacientes com SPP, identificando as queixas relacionadas aos sistemas urinário, fecal e sexual/ginecológico e correlaciona-las com a gravidade e tempo de manifestação da síndrome. Para tanto, baseado no relatório de Avaliação e terminologia das funções e disfunções da musculatura do assoalho pélvico da International Continence Society - ICS ${ }^{24}$ 
foi adaptado pela autora do estudo, o protocolo de Avaliação Fisioterapêutica detalhada, com registros de todos os sintomas relatados, sinais e condições, complementada, quando necessário, com testes adicionais, a fim de orientar uma terapêutica de reabilitação do assoalho pélvico adequada e eficaz para os pacientes com doença neurológica, em especial com SPP.

Como objetivo secundário, foi investigado se 0 protocolo de Avaliação da musculatura do AP, adaptado, era adequado e sensível para identificar as queixas relativas aos sistemas urinário, fecal e sexual/ginecológico em pacientes com doença neurológica, em especial com SPP.

\section{MÉTODO}

\section{Amostra}

Esse estudo de Avaliação da função da musculatura do assoalho pélvico em pacientes com Síndrome Pós Poliomielite é um estudo coorte transversal.

A primeira fase deste estudo ocorreu por contatos telefônicos dos pacientes assistidos no Ambulatório do Setor de Doenças Neuromusculares da UNIFESP, situado no município de São Paulo, Brasil. Em seguida após aprovação do Comitê de Ética Institucional (CEP 1445/05), para os que aceitaram participar da pesquisa, foi esclarecido o Termo de Consentimento Livre e Esclarecido, para ser lido e após assinado, iniciaram-se o Protocolo de avaliação fisioterapêutica. 
A casuística deste estudo constitui-se de 162 pacientes com diagnóstico de Síndrome Pós-Poliomielite, segundo os critérios de Mulder $^{25}$.

Foram incluídos neste estudo 83 pacientes do sexo feminino e do sexo masculino, que apresentavam disponibilidade de deslocamento até à instituição, sendo que 78 desses pacientes aceitaram realizar integralmente a avaliação fisioterapêutica das funções da musculatura do assoalho pélvico.

Foram excluídos pacientes que embora cumprissem os critérios de diagnóstico de SPP, não aceitaram ou puderam participar da pesquisa, tinham dificuldades para agendamento telefônico ou deslocamento até a instituição, apresentavam outras doenças que poderiam ser a causa da nova fraqueza como lesões anteriores, radiculopatias, compressão neuropática e doenças neurológica, ortopédica (exceto escolioses), psiquiátrica ou cirurgias prévias de: câncer (intestinal, próstata, bexiga ou ginecológico), incontinência urinária, incontinência fecal e ginecológica. Também foram excluídos àqueles que não aceitaram realizar a avaliação fisioterapêutica integralmente.

\section{Procedimento}

O instrumento utilizado foi um protocolo de avaliação fisioterapêutica das funções da musculatura do AP, adaptado pela autora do estudo, para pacientes com doença neurológica, especificamente, os diagnosticados com SPP e para cada um dos sexos, baseada no relatório 
de Avaliação e terminologia das funções e disfunções da musculatura do assoalho pélvico da International Continence Society - ICS ${ }^{24}$, complementado com os testes de Avaliação Funcional do Assoalho Pélvico $(A F A)^{17}$ e o de manometria do assoalho pélvico (testes de contração, resistência e relaxamento muscular), acrescido quando necessário de testes adicionais (Estudo Urodinâmico, Ressonância Magnética, Eletro Neuromiografia).

O protocolo de avaliação fisioterapêutico, aplicado pelo mesmo e único profissional, constou de: anamnese; queixas espontâneas relativas aos sistemas urinário, fecal e sexual/ginecológico; história da poliomielite; história da síndrome pós- poliomielite; queixas inquiridas após explicações pertinentes relativas aos sistemas urinário, fecal e sexual/ginecológico; exames clínico específico, Inspeção visual e Palpação do AP, via vaginal e anal; teste subjetivo, Avaliação Funcional do Assoalho Pélvico (AFA), utilizando toque bidigital ou unidigital, para a via vaginal $e$ anal respectivamente, mensurando a contração, sustentação e relaxamento da musculatura do AP; teste objetivo, Manometria do assoalho pélvico (utilizando um probe de pressão, com registros em $\mathrm{mmHg}$ ), mensurando a contração, sustentação e relaxamento da musculatura do AP.

Procedimento do protocolo de avaliação fisioterapêutica das funções da musculatura do assoalho pélvico: o profissional fisioterapeuta apresentou-se ao paciente como profissional que identifica, previne e reabilita as 
funções e disfunções do assoalho pélvico relativas aos sistemas urinário, fecal e sexual/ginecológico; e seguiu o procedimento inicial da avaliação fisioterapêutica, com anamnese e perguntas genéricas a respeito da existência de queixas relativas a esses sistemas (queixas espontâneas). Foi oferecida aos pacientes, aula expositiva tendo como conteúdo os órgãos e o assoalho pélvico, utilizou-se como material de apoio, lâminas de anatomia dos órgãos pélvicos e da musculatura do AP, acompanhadas de explicações a respeito da estrutura e funcionamento dos mesmos. Seguiu-se o protocolo de avaliação com inquirições específicas (queixas inquiridas) para cada um dos sistemas: urinário; fecal e sexual/ ginecológico.

Após coleta de todos os dados pessoais e investigativos, realizou- se o exame clínico específico, que constou de inspeção visual e palpação do assoalho pélvico. Aplicou-se os testes de Avaliação Funcional do Assoalho Pélvico (teste subjetivo) e Manometria do Assoalho Pélvico (teste objetivo).

Durante a anamnese, foram registrados, também, outros sintomas relativos à SPP referidos pelos pacientes.

\section{RESULTADOS}

Dos 83 pacientes elegíveis, foram excluídos 5/83 (um do sexo feminino e quatro do sexo masculino) por se recusarem a realizar o exame clínico específico. A casuística 
teve como menor idade 32 anos e maior idade 72 anos. A média da idade da casuística foi de 49,6 anos, com desvio padrão de 9,87 e intervalo de confiança de $\pm 2,19$, e mediana de 49 anos.

Observou-se que após ataque agudo da pólio houve uma melhora funcional, porém com comprometimento sequelar predominando em membros inferiores. Quanto à caracterização do comprometimento funcional na vigência da SPP, foram raros os pacientes dependentes $(2,6 \%)$, sendo a maioria independente $(97,4 \%)$, cadeirante $(12,8 \%)$ ou não cadeirante $(84,6 \%)$.

\section{Dados temporais da SPP na casuística}

A média de idade da manifestação dos sintomas de SPP foi de 42,5 anos, com desvio padrão de 9,95 anos, e intervalo de confiança de $\pm 2,27$ anos. E a mediana foi de 40 anos. A menor idade da manifestação de SPP foi de 22 anos e a maior idade de 69 anos.

Quanto ao tempo de estabilidade da SPP, a média foi de 35,8 anos, com desvio padrão de 10,98, intervalo de confiança de $\pm 2,61$ anos, e a mediana foi de 35 anos. 0 menor tempo de estabilidade encontrado foi de dez anos e o maior, de 68,0 anos.

Quanto ao tempo de duração da SPP (intervalo de tempo desde a idade em que se instalaram os primeiros sintomas da SPP até idade atual), demonstrado na Tabela 1. O maior percentual de pacientes encontra-se na faixa de tempo de duração da SPP de um a dez anos, com 35 
mulheres $(63,6 \%)$ e 18 dos homens (78,3\%). A média de tempo de instalação da SPP é de sete anos, com desvio padrão de 5,9 anos, e intervalo de confiança de $\pm 1,34$ anos, e a mediana de cinco anos. O menor tempo de duração dos sintomas foi de um ano e o maior, de 27 anos.

Tabela 1. Distribuição dos pacientes em faixas de tempo de duração de SPP por sexo.

\begin{tabular}{lcccc}
\hline Tempo de Duração da & \multicolumn{2}{c}{ Mulheres } & \multicolumn{2}{c}{ Homens } \\
\cline { 2 - 6 } SPP & $\mathbf{N}$ & $\mathbf{\%}$ & $\mathbf{N}$ & $\mathbf{\%}$ \\
\hline $\mathbf{1}$ a 10 anos & 35 & 63,6 & 18 & 78,3 \\
$\mathbf{1 1}$ a 20 anos & 14 & 25,5 & 4 & 17,4 \\
$\mathbf{2 1}$ a 28 anos & 1 & 1,8 & 0 & 0,0 \\
Ignorados & 5 & 9,1 & 1 & 4,3 \\
\hline Total & 55 & 100 & 23 & 100 \\
\hline
\end{tabular}

Os demais sintomas clínicos relatados espontaneamente pelos pacientes com SPP durante a avaliação fisioterapêutica encontram-se na Tabela 2.

Tabela 2. Sintomas clínicos relatados pelos pacientes com SPP durante avaliação fisioterapêutica.

\begin{tabular}{lcc}
\hline Sintomas da SPP & N & \% \\
\hline Dor muscular/articular & 70 & 89,7 \\
Fadiga & 43 & 55,1 \\
Insônia & 18 & 23,1 \\
Alteração respiratória & 14 & 18,0 \\
Alteração ao frio & 12 & 15,4 \\
Quedas constantes & 11 & 14,1 \\
Cafaleia & 7 & 9,0 \\
Depressão & 5 & 6,4 \\
Ganho de peso & 5 & 6,4 \\
Cãibras & 3 & 3,9 \\
\hline
\end{tabular}




\section{Caracterização dos aspectos relacionados à musculatura do assoalho pélvico}

Os sintomas relatados foram coletados para toda casuística, por meio de "queixas espontâneas" ou "queixas inquiridas após explicações pertinentes", para cada um dos sistemas urológico, fecal e sexual/ginecológico relativas às funções da musculatura do assoalho pélvico e encontramse demonstrados nas Tabelas 3 e 4.

Tabela 3. Distribuição de "queixas espontâneas" para cada um dos sistemas urinário, fecal e sexual/ ginecológico, para o grupo das mulheres e o dos homens.

\begin{tabular}{lllll}
\hline \multicolumn{2}{l}{ Queixas Espontâneas } & Mulheres & Homens & Valor de $\boldsymbol{p}$ \\
\cline { 2 - 5 } $\operatorname{sim}$ & $\mathbf{N}$ & 34 & 7 & \\
& $\mathbf{\%}$ & 61,8 & 30,4 & 0,011 \\
\multirow{2}{*}{ Não } & $\mathbf{N}$ & 21 & 16 & \\
\cline { 2 - 5 } & $\mathbf{\%}$ & 38,2 & 69,6 & \\
\hline
\end{tabular}

Tabela 4. Distribuição das "queixas inquiridas após explicações pertinentes" para cada um dos sistemas: urinário, fecal e sexual/ginecológico para o grupo das mulheres e dos homens.

\begin{tabular}{lllll}
\hline \multicolumn{2}{l}{ Queixas Inquiridas } & Mulheres & Homens & Valor de $\boldsymbol{p}$ \\
\multirow{2}{*}{$\operatorname{Sim}$} & $\mathbf{N}$ & 51 & 20 & \\
& $\mathbf{\%}$ & 92,7 & 87,0 & 0,416 \\
\multirow{2}{*}{ Não } & $\mathbf{N}$ & 4 & 3 & \\
\cline { 2 - 4 } & $\mathbf{\%}$ & 7,3 & 13,0 & \\
\hline Total & 55 & 23 & \\
\hline
\end{tabular}

Na investigação criteriosa dessas queixas relativas aos sistemas urinário, fecal e sexual/ginecológico, observou-se que os pacientes tinham dificuldade em relatá-las 
espontaneamente num índice percentual apurado de 61,8\% das mulheres e $30,4 \%$ dos homens. Porém, quando foram oferecidas aos pacientes, aula expositiva tendo como conteúdo a estrutura e função de toda a pelve, da musculatura do AP, do períneo, dos órgãos pélvicos e genitais externos, e depois de realizadas investigações dirigidas, mudanças significativas foram registradas no cenário das queixas, e aqueles índices passaram a 92,7\% das mulheres e $87 \%$ dos homens. A prevalência dos sintomas e sinais pertinentes a essas disfunções apresentou-se associados à Síndrome Pós-poliomielite.

Correlacionando a quantidade de sintomas da SPP com idade atual, tempo de manifestação, tempo de estabilidade, tempo de duração da SPP, Avaliação Funcional do Assoalho Pélvico e manometria, observou-se correlação entre quantidade de sintomas e o tempo de duração da SPP, indicando que quanto maior o tempo de duração da SPP, maior é a quantidade de sintomas, demonstrado na Tabela 5.

\section{DISCUSSÃO}

\section{Etiologia, fisiopatologia e diagnóstico}

Os sintomas da SPP e seus índices de ocorrência não estão definitivamente delineados na literatura médica. As dificuldades encontradas são o reflexo de uma multiplicidade de fatores limitativos vivenciados pelos profissionais da saúde e presentes nos estudos etiológicos, 
ainda não claramente conclusivos, nos critérios de diagnóstico, em que há áreas nebulosas, em terapêuticas, cuja magnitude dos efeitos para um razoável número delas não foi comprovada em definitivo.

Tabela 5. Matriz da correlação da quantidade de sintomas.

\begin{tabular}{|c|c|c|c|}
\hline & & \multicolumn{2}{|c|}{ Quantidade de Sintomas } \\
\hline & & Correlação & Valor de $p$ \\
\hline \multicolumn{2}{|c|}{ Idade atual } & $-4,1$ & 0,719 \\
\hline \multicolumn{2}{|c|}{ Tempo de Manifestação da SPP } & $-16,5$ & 0,161 \\
\hline \multicolumn{2}{|c|}{ Tempo de Estabilidade } & $-21,7$ & 0,078 \\
\hline \multicolumn{2}{|c|}{ Tempo de duração SPP } & 30,2 & 0,009 \\
\hline \multirow{3}{*}{ Anal } & AFA & 17,1 & 0,367 \\
\hline & Força & $-9,9$ & 0,602 \\
\hline & Resistência & $-7,5$ & 0,695 \\
\hline \multirow{3}{*}{ Vaginal } & AFA & 1,7 & 0,910 \\
\hline & Força & 23,7 & 0,104 \\
\hline & Resistência & 20,3 & 0,166 \\
\hline \multirow{3}{*}{ Geral } & AFA & 6,1 & 0,594 \\
\hline & Força & 5,6 & 0,627 \\
\hline & Resistência & 3,0 & 0,795 \\
\hline
\end{tabular}

Dependentemente do conceito de SPP a ser considerado, a SPP ocorre em até $70 \%$ dos sobreviventes da poliomielite paralítica ${ }^{4}$.

Em estudo epidemiológico realizado, no Setor de investigação de doenças Neuromusculares da UNIFESP, $77,2 \%$ de uma casuística de 167 pacientes apresentavam $\mathrm{SPP}^{26}$.

No Brasil, os principais obstáculos ao conhecimento da SPP são provavelmente a ausência de diagnóstico e sua inexatidão. A desinformação a respeito da SPP por parte da 
classe médica e dos indivíduos acometidos por ela é uma explicação para isso. Só muito recentemente foram divulgadas as primeiras referências à SPP no país ${ }^{4,26,27}$, fato justificado pelo número crescente de indivíduos com sequela de poliomielite paralítica que procuram serviços especializados de neurologia e que estão mais cientes graças à divulgação mais ampla e frequente permitida pela globalização e pelo fácil acesso à internet.

\section{Característica da população estudada}

A frequência predominante do sexo feminino apurada em nosso estudo é semelhante à muitos outros, foi observado um interesse maior por parte das mulheres em relação à saúde. Elas conversam mais entre si, aceitam e buscam ajuda médica ${ }^{12-14,28,29}$.

A média de idade de manifestação dos sintomas de SPP foi de 42,5 anos e a mediana de 40 anos. A idade mais baixa foi de 22 anos e a mais avançada, 69 anos.

O aparecimento de novos sintomas, prevalecendo nas idades da quarta e quinta décadas de vida, confirmou sua relação direta com a fisiopatologia da SPP, em detrimento do fator envelhecimento. Apresentavam idade inferior a 60 anos, quando do aparecimento de novos sintomas, 93,6\% dos pacientes, o que corrobora a ideia de que as manifestações clínicas não podem ser relacionadas a perdas motoras causadas pela idade.

Quanto ao platô de estabilidade (período de latência que se segue após o período de recuperação da pólio), 
nossa pesquisa encontrou como menor tempo dez anos e maior 68 anos, tendo como média 35,8 anos e mediana 35 anos. Assim, há semelhança e pouca divergência significativa com vários estudos: 30 a 40 anos ao platô de estabilidade $^{8}$; outro em que havia um platô variando de 25 a 30 anos $^{6}$; alguns outros pesquisadores, preferem a menção a um tempo genérico, p.e., "anos"9-12. As dificuldades e os erros na determinação do diagnóstico de SPP, na coleta de dados e no fornecimento destes pelos próprios pacientes, e na apuração da data de início de ocorrência de novos sintomas são fatores prováveis para se explicar os diferentes platôs de estabilidade.

Nossa pesquisa confirma também os achados da literatura atinentes à diversidade de topografia afetada no ataque agudo do poliovírus no período após a recuperação da pólio e na vigência da SPP. Em nosso estudo, a topografia no ataque agudo do poliovírus mostra $100 \%$ de afetação dos membros inferiores. Foi reconhecida elevada proporção de agravamento dos sintomas na vigência da SPP, levando à necessidade de auxílio para locomoção em muitos $\operatorname{casos}^{30}$.

\section{Características clínicas da SPP}

Quanto aos aspectos clínicos, a literatura lista como sintomas prevalentes de SPP a nova fraqueza muscular, fadiga, dores muscular e articular ${ }^{4,6,12,28,29}$ e como os de frequência menor a intolerância ao frio, distúrbios do sono, fasciculações, nova atrofia, ganho de peso, dificuldades 
respiratória e de deglutição (disfagia) ${ }^{3,9,28}$. O mesmo foi encontrado em nosso estudo. Apesar de nossa pesquisa objetivar a investigação detalhada dos sintomas urinários, fecais e sexuais/ginecológicos, esses sintomas foram registrados após os pacientes referirem espontaneamente.

\section{SPP x Alteração da musculatura do assoalho pélvico}

A SPP determina a aparição de novos sintomas, como nova fraqueza e fadiga, que podem comprometer músculos funcionalmente não afetados anteriormente, atrofia muscular residual e arreflexia, incidentes em pelo menos um membro, preservada a sensibilidade ${ }^{3,4}$ e a função esfincteriana ${ }^{4}$, e deterioração da habilidade funcional para as atividades da vida diária, mobilidade e função dos membros.

Segundo a International Continence Society $(\text { ICS })^{24}$, a avaliação da função e disfunção da musculatura do assoalho pélvico deve proceder à análise criteriosa, que inclui sintomas, sinais e condição.

Sintomas são qualquer fenômeno ou mudança provocada no organismo por uma doença, e que, observados por meio de sinais, ou descritos pelo paciente, permitem estabelecer um diagnóstico. Podem ser relatados espontaneamente ou respondidos em questionário ou após inquirição na anamnese. Em geral percebidos pelo paciente, acompanhante ou cuidador, levá-lo a procurar um profissional da saúde.

Os sinais são observados pelo examinador e permitem 
a verificação e quantificação de sintomas. Podem ser testados no exame físico, como inspeção visual, palpação e testes específicos, p.e., a capacidade de contração, sustentação da contração e relaxamento dos músculos do assoalho pélvico.

Durante o exame clínico, todos os procedimentos realizados foram semelhantes aos estudos da literatura ${ }^{17-21}$.

É ampla a literatura em estudos a respeito da função e disfunção da musculatura do assoalho pélvico que apontam para uma grande prevalência de alterações morfofuncionais dos sistemas urinário, fecal, sexual/ginecológico ${ }^{17-22}$. No entanto, são escassos os estudos que abordam a função e disfunção da musculatura do assoalho pélvico em pacientes com doença neurológica, em especial, aqueles com SPP. Em pouquíssimos estudos encontrados na literatura ${ }^{13-16}$, constam a prevalência de sintomas urinários, gastrintestinais, sexuais e ginecológicos, contudo eles concordam relativamente com os achados de nossa pesquisa. Essa relatividade deveu-se principalmente à utilização de uma coleção mais restrita de dados (exclusivamente coletados por meio de questionários) por parte dos demais pesquisadores. Os resultados apurados em nossa pesquisa apresentam semelhanças aos poucos trabalhos encontrados da literatura no tocante às queixas espontâneas (sintomas), das disfunções da musculatura do assoalho pélvico.

A experiência com esses 78 pacientes com SPP levounos ao reconhecimento da importância de uma ação 
fisioterapêutica no cuidado com o assoalho pélvico, iniciando com uma identificação e registro de todos os sintomas relatados espontaneamente ou após inquirição, sinais e condições que aflige o paciente com doença neurológica em especial os com diagnostico de SPP, a fim de orientar uma terapêutica de reabilitação, segura e eficaz.

É possível que os pacientes influenciados por condutas e posturas sociais encontrem dificuldades em relatar espontaneamente suas queixas, seja por vergonha, ou timidez, ou até mesmo por não as julgarem importantes, sendo assim reínteramos a importância de uma investigação dirigida.

\section{CONCLUSÃO}

Pacientes com síndrome pós-poliomielite apresentam comprometimento significante da musculatura do assoalho pélvico com alterações nas funções urinárias, fecais, sexuais/ginecológicas semelhante ao que ocorre com os demais músculos esqueléticos acometidos, sendo que quanto maior for a fraqueza e o tempo de manifestação da síndrome pós-poliomielite mais grave é o comprometimento.

Em relação ao objetivo secundário, o protocolo de avaliação fisioterapêutica para as funções da musculatura do assoalho pélvico, baseada nas recomendações da International Continence Society $(\text { ICS })^{(24)}$, mostrou- se um 
instrumento adequado e sensível para avaliar e identificar disfunções dos sistemas urinário, fecal, sexual/ginecológico em pacientes com doença neurológica, em especial os com síndrome pós- poliomielite, devido principalmente à correlação entre as "queixas espontâneas" e as "queixas inquiridas" após explicações pertinentes.

\section{REFERÊNCIAS}

1.Raymond M. Paralysie essentielle de l'enfance, atrophie musculaire consecutive. C R Soc Biol 1875;27:158-60.

2.Dalakas MC, Elder G, Hallet M, Ravits J, Baker M, Papadopoulos N, et al. A long-term follow-up study of patients with post-poliomyelitis neuromuscular symptoms. New Engl J Med 1986;314:959-63. http://dx.doi.org/10.1056/NEJM198604103141505

3.Kimberley C, Dinsmore S, Grafman J, Dalakas M. A personality profile of pactients diagnosed with post-polio syndrome. Neurology 1994;44:1809-11. http://dx.doi.org/10.1212/WNL.44.10.1809

4.Oliveira ASB, Maynard FM. Síndrome pós-poliomielite: aspectos neurológicos. Rev Neurocienc 2002;10:31-4.

5.Borg K, Borg J, Edstrom L, Grimby L. Effects of excessive use of remaining muscle fibers in prior polio and LV lesion. Muscle Nerve 1988;11:1219-30. http://dx.doi.org/10.1002/mus.880111206

6.Dalakas MC. The post-polio syndrome as an evolved clinical entity. Definition and clinical descripition. Ann NY Acad Sci 1995;753:68-80.

7.Agre JC, Rodriguez AA,Tafel AJ. Late effects of polio: critical review of literature on neuromuscular function. Review article. Arch Phys Med Rehabil 1991;72:923-31. http://dx.doi.org/10.1016/00039993(91)90013-9

8.Trojan DA, Cashman NR. Pathophysiology and diagnosis of postpolio syndrome. NeuroRehabilitation 1997;8:83-92. http://dx.doi.org/10.3233/NRE-1997-8203

9.Howard RS. Poliomyelitis and the postpolio syndrome. BMJ 2005;330:1314- 8. http://dx.doi.org/10.1136/bmj.330.7503.1314 10.Bouza C, Amate JM. Post polio-syndrome: a review of its clinical characteristics and treatment. Rev Neurol 2006;43:295-301.

11.Schanke AK, Stanghelle JK. Fatigue in polio survivors. Scientific Review. Spinal Cord 2001;39:243-51.

12.Agre JC, Grimby G, Rodriguez AA, Sperling KB. Symptoms and clnical impressions of patients seen in a postpolio clinic. Arch Phys Med Rehabil 1989;70:367-70.

13. Bozeman T, Anuras S. Gastrointestinal manifestations in the postpólio syndrome (abs). Am J Gastroenterol 1991;86:1367. 
14.Jonhson VY, Hubbard D, Vordermark JS. Urologic manifestations of postpolio syndrome. J Wound Ostmy Continc Nurs 1996;23:21823. http://dx.doi.org/10.1016/S1071-5754(96)90094-7

15. Howard RS, Wiles CM, Spencer GT. The late sequelae of poliomyelitis. Q J Med 1988;66:219-32.

16.Chansens ER, Umlauf M, Valappil T, Singh KP. Nocturnal problems in postpolio syndrome: sleep apnea symptoms and nocturia. Rehabil Nurs $\quad 2001 ; 26: 66-71 . \quad$ http://dx.doi.org/10.1002/j.20487940.2001.tb01928.x

17. Ortiz CO, Coya NF Ibañez G. Evaluacion functional del piso femenino (Classificacion Funcional). Bol Soc Latinoam Uroginecol Cir Vaginal 1996;1:5-9.

18. Isherwood $\mathrm{PJ}$, Rane A. Comparative assessment of pelvic floor strength using a perineometer and digital examination. $\mathrm{Br}$ J Obstet Gynaecol 2000;107:1007-11. http://dx.doi.org/10.1111/j.14710528.2000.tb10404.x

19.Bo K, Sherburn M. Evaluation of female pelvic-floor muscle function and strength. Phys Ther 2005;85:269-82.

20. Oliveira C, Lopes MAB, Pereira LCL, Zugaib M. Effects of pelvic floor muscle training during pregnancy. Clinics (Sao Paulo) 2007;62:439-46.

http://dx.doi.org/10.1590/S1807-

$\underline{59322007000400011}$

21.Chen X, Gong Y, Wu D, Li X, Li H, Tong X, et al. Pre- and postoperative Evaluation of Pelvic Floor Muscle Function in POP Patients Using Surface Eletromyography and Digital Palpation. Neurourol Urodyn 2014;33:403-7.

http://dx.doi.org/10.1002/nau.22430

22.Corona-Quintanilla DL, Zempoalteca R, Arteaga L, Castelán F, Martínez-Gómez M. The Role of Pelvic and Perineal Striated Muscles in Urethral Function During Micturition in Female Rabbits. Neurourol Urodyn 2014;33:455- 60. http://dx.doi.org/10.1002/nau.22416

23. Moore Kl. Anatomia orientada para clínica. $3^{a}$ ed. São Paulo: Guabanara Koogan, 1992, p. 263-79.

24. Messelink B, Benson T, Berghmans B, Bø K, Corcos J, Fowler C, et al. Standardization of terminology of pelvic floor muscle function and dysfunction: Report from the pelvic loor clinical assessment group of the International Continence Society. Neurourol Urodyn 2005;24:374-80. http://dx.doi.org/10.1002/nau.20144

25. Mulder DW, Rosenbaum RA, Layton DD. Late progression of poliomyelitis or forme fruste amyotrophic lateral sclerosis? Mayo Clin Proc 1972;47:756- 61.

26.Quadros AAJ. Síndrome pós polio: uma nova doença velha (Tese). São Paulo: Universidade Federal de São Paulo, 2005, 233p.

27. Conde MTRP. Síndrome pós-poliomielite: aspectos epidemiológicos e prognósticos (Tese). São Paulo: Universidade Federal de São Paulo, 2007, 124p.

28. Halstead LS, Wiechers DO, Rossi CD. Late effects of poliomyelitis. Part II: Results of survey of 201 survivors. South Med J $1989 ; 78: 1281-7$. 
29.Farbu E, Gilhus NE, Bames MP, Borg K, De Visser M, Driessen A, et al. EFNS guideline on diagnosis and management of post-polio syndrome. Report of an EFNS task force. Eur J Neurol 2006;13:795801. http://dx.doi.org/10.1111/j.1468-1331.2006.01385.x

30.Ramlow J, Alexander M, La Porte R, Kaufmann C, Kuller L. Epidemiology of post-polio syndrome. Am J Epidemiol 1992;136:76986. http://dx.doi.org/10.1093/aje/136.7.769 